\title{
Weather and Climate Manipulation as an Optimal Control for Adaptive Dynamical Systems
}

\author{
Sergei A. Soldatenko \\ St. Petersburg Institute for Informatics and Automation, The Russian Academy of Sciences, No. 39, 14th Line, \\ St. Petersburg 199178, Russia \\ Correspondence should be addressed to Sergei A. Soldatenko; soldatenko@iias.spb.su
}

Received 30 July 2016; Accepted 13 November 2016; Published 12 January 2017

Academic Editor: Dimitri Volchenkov

Copyright (C) 2017 Sergei A. Soldatenko. This is an open access article distributed under the Creative Commons Attribution License, which permits unrestricted use, distribution, and reproduction in any medium, provided the original work is properly cited.

The weather and climate manipulation is examined as an optimal control problem for the earth climate system, which is considered as a complex adaptive dynamical system. Weather and climate manipulations are actually amorphous operations. Since their objectives are usually formulated vaguely, the expected results are fairly unpredictable and uncertain. However, weather and climate modification is a purposeful process and, therefore, we can formulate operations to manipulate weather and climate as the optimization problem within the framework of the optimal control theory. The complexity of the earth's climate system is discussed and illustrated using the simplified low-order coupled chaotic dynamical system. The necessary conditions of optimality are derived for the large-scale atmospheric dynamics. This confirms that even a relatively simplified control problem for the atmospheric dynamics requires significant efforts to obtain the solution.

\section{Introduction}

Weather and climate manipulation is defined as a deliberate intervention in the natural evolution of physical processes in the atmosphere and other components of the earth's climate system (ECS) in order to achieve some desired results $[1,2]$. Here, as usual, the ECS $(\mathcal{S})$ is understood as a complex largescale physical system that consists of five basic and interacting constituent complex subsystems, namely [3],

(i) atmosphere $(\mathscr{A})$, the gaseous and aerosol envelope of the earth that propagates from the land, water bodies, and ice-covered surface outward to space,

(ii) hydrosphere $(\mathscr{H})$, the oceans and other water bodies on the surface of our planet and water that is underground and in the atmosphere,

(iii) cryosphere $(\mathscr{C})$, the sea ice, freshwater ice, snow cover, glaciers, ice caps and ice sheets, and permafrost,

(iv) lithosphere $(\mathscr{L})$, the solid, external part of our planet,

(v) biosphere $(\mathscr{B})$, the part of our planet where life exists; that is,

$$
\mathcal{S}=\mathscr{A} \cup \mathscr{H} \cup \mathscr{C} \cup \mathscr{L} \cup \mathscr{B} \text {. }
$$

Each component of the ECS is characterized by a finite set of variables, usually called state variables, whose values at a given time determine the instant state of the ECS. All components of the ECS are extremely complex physical systems that in turn also are composed of constituent elements (subsystems). It should be noted that the current status of climate system studies is characterized by dramatic progress achieved over the last few decades in understanding basic physical processes and fundamental feedback mechanisms that control the climate formation and change (thanks to the advances in computing hardware and algorithms and due to a substantial increase in the volume of climatological data [4$8])$. The detailed retrospective analysis of climate science can be found, for example, in [9]. However, our knowledge for climate remains incomplete due to the enormous complexity of the ECS. A deeper understanding of current climate, its variability, and change is among the greatest challenges facing modern science that can help not only to project the future climate but also to develop methods for controlling the ECS.

The objectives and methods of weather modification are distinctly different than those of climate manipulation. This distinction is due to the fact that the terms "weather" and "climate" have different meanings. Weather is defined as the 
daily conditions of the atmosphere in terms of such atmospheric variables as temperature, humidity, wind direction and velocity, surface pressure, clouds, and precipitation. In contrast, climate represents the ensemble of states traversed by the climate system over a sufficiently long enough temporal interval. Here, the ensemble means not only the set of system states, but also a probability measure defined on this set. Climate, roughly speaking, can be considered as the "average" weather, in terms of mean and variance, in a certain geographical location over many years.

Weather modification is aimed at altering weather conditions and weather phenomena locally or regionally. The successful examples of weather modification operations are cloud seeding to increase precipitations, the removal of fogs, stratiform and convective clouds, the suppression of hail, and lightning (e.g., [10-14]). The large-scale weather modification experiments such as the reduction of the intensity of hurricanes were less successful [15-17]. Thus weather modification aims to intentionally affect processes and phenomena only in the atmosphere, the most volatile component of the ECS. Climate manipulation known also as a climate engineering or geoengineering was suggested as a response measure on the global climate change that currently occurs on our planet [3]. Geoengineering aiming at stabilizing the global climate and preventing further warming is a planetary-scale intentional intervention on the ECS and represents a multidisciplinary problem of extreme complexity, which is currently explored mostly theoretically because the consequences and unintended side effects of climate manipulation operations are very uncertain. Geoengineering requires further consideration of not only scientific and technical issues, but also the legal and ethical aspects and limitations. It is expected that the interest in climate and weather manipulation will continue to grow, so that it makes it necessary to develop the appropriate theoretical framework. To date a number of methods and technologies were proposed by scientists and engineers to stabilize the ECS and to mitigate the global warming (e.g., [18-24]).

The realization of both weather modification and climate engineering operations is a goal-directed process, having specific objectives that should be somehow formulated and certainly achieved. Mathematically, weather and climate manipulation can be viewed as an optimization problem $[25,26]$. Consequently, instead of examining weather and climate manipulation as amorphous actions without clearly defined objectives, boundaries, and methods, we can consider operations to modify weather and climate as an optimal control problem for the ECS and its components.

It is important to make the following comments concerning controllability of the ECS and its components and, in particular, the atmosphere. Controllability can be thought of as a property of a control system that indicates the ability to transfer a system from one state to another, using admissible operations (e.g., [27]). From the perspective of physics, the ECS is a controllable system, and its key driver is the incoming shortwave solar radiation. On the geological time scale, the earth's climate has been highly variable throughout history as a result of both external forcing and internal perturbations [28] (e.g., variations in the chemical and aerosol composition of the atmosphere and aperiodic El Niño-Southern Oscillation). Since the incoming solar radiation can be viewed as a primary control variable, geoengineering seeks to reduce global warming by using physically feasible ability to manipulate the climate via changes in the amount of incoming solar radiation (by varying reflection or absorption) as well as natural feedback mechanisms that exist in the ECS. However, geoengineering is not the same as control, and thus the term "engineering" rather than "control" is currently used in the literature to emphasize this difference. Considering geoengineering within the formal framework of the optimal control theory, we are faced with a number of very hard mathematical problems, such as the proof of the controllability property for any particular climate model.

Similar problems arise when we consider weather modification. For example, we can create the artificial precipitation by cloud seeding; however the formal proof of the existence of the optimal solution and the proof of the controllability property for an applied mathematical model are problems of extreme difficulty. For this reason, in weather modification, the term "intentional (deliberate) action" is usually used instead of "control." Thus, we have to make the distinction between control as an engineering problem and control as a mathematical problem.

In this paper, the weather and climate manipulation is examined as an optimal control problem for the climate system, which is considered as a complex adaptive dynamical system. The complexity of the climate system is discussed and illustrated using the simplified low-order coupled chaotic dynamical system. Finally, as an example, the necessary conditions of optimality are derived for the large-scale atmospheric dynamics. This shows that even a relatively simplified control problem for the atmospheric dynamics requires significant efforts to obtain the solution.

\section{Complexity in the Earth's Climate System}

Let us make some preliminary comments. The term "system" generally refers to a goal-oriented set of interconnected and interdependent elements that operate together to achieve some objectives [29]. The system is called complex if it possesses such characteristics as emergent behavior, nonlinearity and high sensitivity to initial conditions and/or to perturbations, self-organization, chaotic behavior, feedback loops, spontaneous order, robustness, hierarchical structure, and some others [30]. Complexity in systems appears in particular from spatiotemporal interactions between their components. These nonlinear interactions lead to the appearance of new dynamical properties (e.g., synchronous oscillations and other structural changes) that cannot be observed by exploring constituent elements individually. Let us go one step forward. Complex systems include a special class of systems that have the capacity to adopt to system's environment. These systems are known as complex adaptive systems (e.g., $[31,32])$. In complex adaptive system its parts are linked together in such a way that the entire system as a whole has the capacity to transform fundamentally the interrelations and interdependences between its components, the collective 
behavior of a system, and also the behavior of individual components due to the external forcing. Complex adaptive systems are dynamical systems since they evolve and change over time. These systems have a number of properties that include the following [32, 33]: coevolution, connectivity, suboptimality, requisite variety, iteration, edge of chaos and, certainly, emergence, and self-organization.

The ECS is a large-scale and peculiar dynamical system that possesses numerous unique physical, dynamical, and chemical properties (e.g., $[7,8,33])$. The study and a fortiori control of the ECS are a highly difficult problem. There is no doubt that the ECS can be viewed as a complex adaptive system. This is because of the following.

(i) The ECS is a complex system combining the atmosphere, hydrosphere, cryosphere, land, and biota together with global biochemical cycles (first of all, cycles of $\mathrm{CO}_{2}, \mathrm{~N}_{2} \mathrm{O}$, and $\mathrm{CH}_{4}$ ) and aerosols. The climate system's components are heterogeneous thermodynamical systems characterized by specific variables that determine their states. Subsystems of the ECS have strong differences in their structure, dynamics, physics, and chemistry. They embrace processes with different temporal and spatial scales and link together via numerous coupling physical mechanisms, which can be weak and strong. Each constituent component of the ECS is also a complex and possibly also adaptive system. For example, the following weather systems could be viewed as subsystems of an overarching atmospheric system: tropical cyclones, hurricanes, mid-latitude cyclones and anticyclones, clouds, precipitations, and so forth.

(ii) The ECS has a hierarchical structure. Each component of the ECS can be characterized by a specific response time. The belonging of a certain component to the ECS is determined by the ratio between the temporal scale of processes under consideration and the response time of the ECS components. For example, the atmosphere, which has a response time of about one month in the troposphere, can be considered as a sole component of the ECS for processes with temporal scales of days to weeks. In this case, oceans, land surface, and ice cover are considered as the boundary conditions and/or external forcing. If we study processes which have temporal scales of months to years, the atmosphere and ocean must be included in the ECS together with sea ice.

(iii) The ECS has a large number of positive and negative feedback mechanisms, for example, ice-albedo feedback (positive feedback), water vapor feedback (positive feedback), cloud feedback (both positive and negative feedbacks), carbon cycle feedback (negative feedback), and feedback due to Arctic methane release (positive feedback).

(iv) Physical and dynamical processes in the ECS cover a broad spectrum of temporal and spatial scales. Time scales are varied from seconds to decades, and spatial spectrum of dynamical processes covers molecular to planetary scales. Dynamical processes in the ECS and its components are nonlinear. Subsystems of the ECS interact with one another nonlinearly producing under certain conditions a chaotic behavior of subsystems and the overall climate system.

(v) The ECS and its components inherently have emergent properties. Examples of atmospheric emergent phenomena include but not limited to clouds, largescale eddies (cyclones and anticyclones), and smallscale vortices such as tornados. Examples of climate emergent phenomena are the El Niño-Southern Oscillation, which is quasiperiodic irregular variation in ocean surface temperature over the Pacific in tropics that strongly influences the global climate and ocean circulation patterns. Natural emergent phenomena appear spontaneously under certain favorable conditions.

(vi) The ECS is thermodynamically open and nonisolated system because it exchanges energy with its surroundings. However, the ECS is a closed system for the exchange of matter with outer space. The energy that drives the ECS is a solar energy. The ECS is affected by changes in external driving forces, which imply natural causes such as solar activity variations and volcanic activities and human-made changes in chemical composition of the atmosphere. However, the impact of the ECS on the outer space is insignificant. Currently, changes in climate are mostly affected by variations in the atmospheric composition of particles and gases. In the Arctic the role of changes in albedo (reflection coefficient) is also tangible. In spite of the fact that the most significant heat-trapping gas is atmospheric water vapor, the most influential gas component to affect the climate change is $\mathrm{CO}_{2}$, which comprises about 70 percentage of points of the global warming potential [3].

(vii) The components of the ECS are also nonisolated systems. They act as cascading systems and interact with each other in various ways including the transfer of momentum, sensible and latent heat, gases, and particles. All together they compose the climate system, which is a unique large-scale natural system.

(viii) Dynamical processes in the ECS periodically and irregularly oscillate due to both internal factors (natural oscillations) and external forcing (forced oscillations). Natural fluctuations are due to internal instability (e.g., convective, barotropic, and baroclinic instabilities) with respect to stochastic perturbations. Human impacts, both intentional and unintentional, are considered as external forcing.

Certainly, there are other specific properties of the ECS that should be taken into account while studying climate as a complex adaptive system.

With regard to weather and climate manipulation, it is necessary to underline that the response of nonlinear systems to external perturbations is completely different, both quantitatively and qualitatively, than the response of linear 
systems [34]. Recall that operations to manipulate weather and climate represent external forcing with respect to the ECS. This is very important in terms of assessing the effects of weather and climate manipulation operations.

To study and simulate the ECS we should assign some mathematical object that is an abstract representation of the real climate system. This object is usually known as an "ideal" model of the system of interest. The climate system model represents a set of interacting and interdepending subsystems (agents). The number of these subsystems is determined by the objectives of problem under consideration. For example, to study the large-scale climate variability the model can include the following major agents: tropical, mid-latitude and polar troposphere, stratosphere, land ice, oceans and sea ice, surface and boundary layers, hydrological cycle, clouds of various types, precipitations, aerosols, $\mathrm{CO}_{2}$ and $\mathrm{CH}_{4}$ cycles, solar radiation, and terrestrial emission. Other subsystems of the ECS (e.g., vegetation, land surface, and biota) can be considered as the boundary conditions and external forcing.

The major components of the ECS are physical continuum and their evolution can mathematically be described by a set of multidimensional nonlinear differential equations in partial derivatives:

$$
\begin{aligned}
\partial_{t} \varphi(r, t) & =\mathscr{M}(\varphi(r, t), \alpha(r, t)), \\
\varphi(0, t) & =\varphi_{0}(r),
\end{aligned}
$$

where $\varphi$ is a state vector of a system, $\alpha$ is a parameter vector, $r \in D \subset \mathbb{R}^{3}$ is a vector of spatial variables, $t \in$ $[0, \mathscr{T}]$ is the time, $\mathscr{M}$ is a nonlinear differential operator that describes the evolution of a system, and $\varphi_{0}$ is initial conditions. Since (2) describes a continuous medium, the state vector $\varphi$ is infinite-dimensional. The solution to such infinite-dimensional system cannot be found analytically, and we need to employ numerical methods. To obtain numerical solution, the original set of (2) is replaced with discrete spatiotemporal approximations using some appropriate technique (e.g., finite-difference method and Galerkin approach). It is very important to underline that a large number of physical processes and cycles (model agents), which also may be viewed as a system, cannot be explicitly represented in the climate model due to its discrete spatiotemporal structure. State-of-the-art models of the ECS are generally unable to realistically simulate processes on spatial scales of the order of twice the model grid length [35]. Such thermodynamical, physical, and chemical processes and cycles are parameterized, that is, expressed parametrically using simplified description. Some of these newly introduced parameters can be considered as controls (see below). By varying control parameters, we can formally manipulate the ECS and its components.

Models of the ECS due to their extreme complexity require extensive computational resources to study the behavior of climate system and its components by computer simulations. However, in order to mimic some features of the ECS behavior and to study its certain properties as a complex system we can use simplified low-dimensional models. For illustrative purposes, we consider a coupled nonlinear model, which is composed of fast (the "atmosphere") and slow ("the ocean") components and obtained by coupling of two versions of the original Lorenz system [36] with distinct temporal scales (e.g., [37-39]):

$$
\begin{aligned}
& \frac{d x}{d t}=\sigma(y-x)-c(a X+k), \\
& \frac{d y}{d t}=r x-y-x z+c(a Y+k), \\
& \frac{d z}{d t}=x y-b z+c_{z} Z, \\
& \frac{d X}{d t}=\varepsilon \sigma(Y-X)-c(x+k), \\
& \frac{d Y}{d t}=\varepsilon(r X-Y-a X Z)+c(y+k), \\
& \frac{d Z}{d t}=\varepsilon(a X Y-b Z)-c_{z} z .
\end{aligned}
$$

Here lower case and capital letters represent, respectively, the "atmosphere" (fast model) and the "ocean" (slow model); $\sigma, r$, and $b$ are the parameters of Lorenz system; $c$ and $c_{z}$ are parameters that describe the coupling strength between fast and slow models, $a$ is the amplitude scale factor, $k$ is an "uncentering" index, and $\varepsilon$ is the time-scale separation parameter. For simplicity but without loss of generality we can assume that $a=1, k=0$, and $c=c_{z}$. The combination of two Lorenz systems allows one to imitate the interaction between fast-oscillating atmosphere and slow-fluctuating ocean [40, 41].

The Lorenz system is one of the most well-known nonlinear dynamical systems, which derived from the equations of atmospheric Rayleigh-Bénard convection [42]. Three variables of the Lorenz model, $x, y$, and $z$, are, respectively, the intensity of convective motion and horizontal and vertical temperature gradients. The model parameters have the following meanings: $\sigma$ is the Prandtl number, $r$ is a normalized Rayleigh number, and $b$ is a nondimensional wavenumber. Properties of the Lorenz model are well known and described in the literature (e.g., [43]). We only note that the original Lorenz system can be studied using different values of parameters. However, for the standard parameter values $\sigma=10, b=8 / 3$, and $r=28$, the Lorenz model exhibits chaotic behavior and possesses a two-wing strange attractor, which has a fractal dimension of about $2.06[36,43]$. For $\sigma=10$ and $b=8 / 3$, the chaotic behavior is detected if $r>r_{c}$, where $r_{c} \approx 24.74$. Varying the parameter $r$, we can observe structural changes in the system dynamics. In our computations the standard values of parameters were used. We also assume that $\varepsilon=0.1$.

The dynamics of coupled system (3) strongly depends on the parameter $c$. This parameter, referred to as the coupling parameter, describes the interaction degree between the fast and slow systems [44]. In the existing climate models, atmosphere and ocean are commonly coupled by adjusted airsea fluxes of heat, momentum, and fresh water using various parameterization techniques (e.g., [45]). In this context, the parameter $c$ can be viewed as a simple parameterization of the 


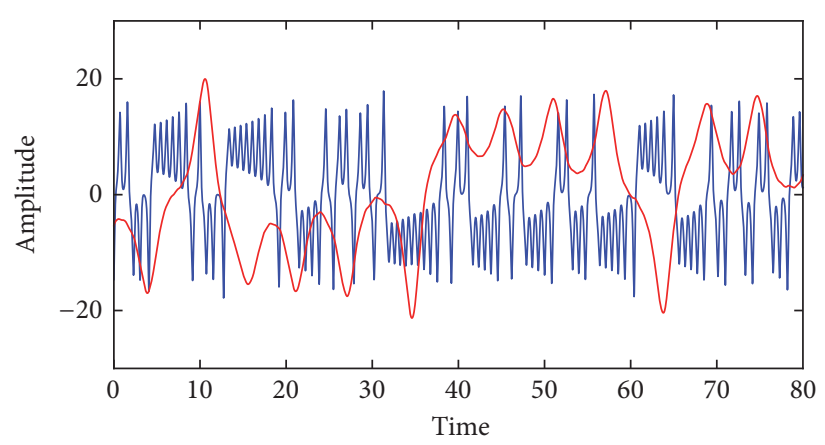

$x$
$-X$
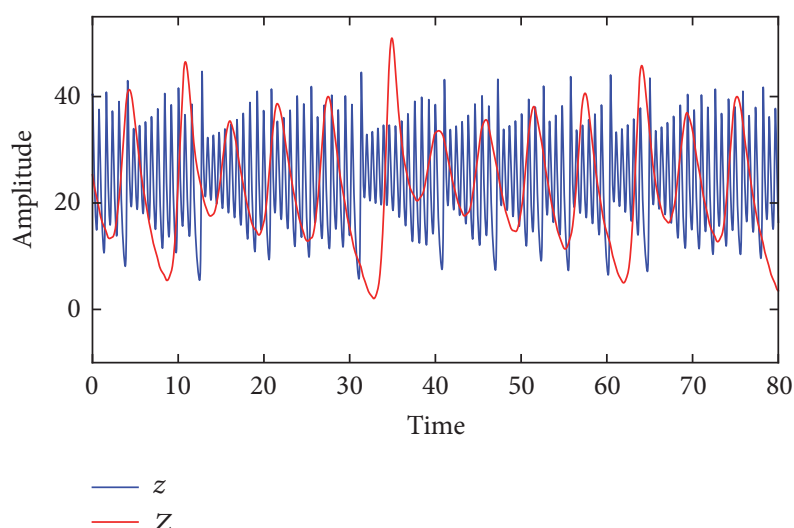

(a)

(b)

Figure 1: Fast ( $x$ and $z$ ) and slow $(X$ and $Z$ ) variables for the case of $c=0.15$.

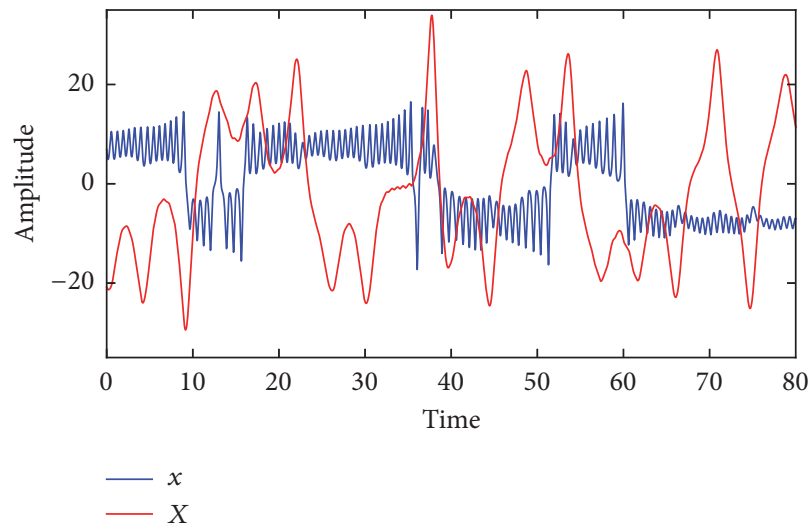

(a)

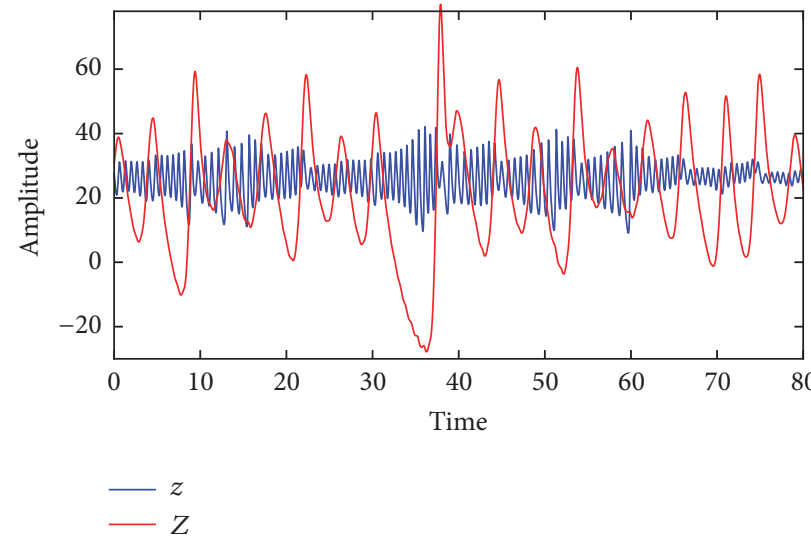

(b)

Figure 2: Fast $(x$ and $z$ ) and slow $(X$ and $Z$ ) variables for the case of $c=0.5$.

interaction between two models used in this study. Figures 14 show the temporal changes of fast $(x$ and $z$ ) and slow $(X$ and $Z$ ) variables calculated by the numerical integration of the coupled model equations. Even a cursory examination of these figures reveals that when a coupling strength parameter is varied, the qualitative behavior of the system changes. Qualitative shifts in the dynamical properties of coupled system can be traced by the spectrum of conditional Lyapunov exponents. These exponents, on the one hand, characterize the average rate of exponential divergence of nearby orbits in the phase space, and, on the other hand, they are used to analyse the synchronization with coupled systems [44]. When the coupling strength parameter tends to zero, coupled system (3) has six distinct Lyapunov exponents: two positive, two negative, and two zero. The dependence of two largest Lyapunov exponents on the parameter $c$ is shown in Figure 5 [39]. These exponents are monotonically decreasing functions. Being initially positive, they approach the $x$-axis at about $c \approx 0.8$ and at about $c \approx 0.95$ become negative. Thus, the phase-synchronous regime is observed when $c>0.95$.
When $c>1.0$, the system approaches a limit cycle since all six Lyapunov exponents become negative.

However, the real climate system is a forced system. To explore the influence of external forcing (constant, periodic, aperiodic, stochastic, etc.) on the coupled system dynamics one can add additional terms in the right-hand sides of model equation (3) (e.g., [46]). This allows the testing of various strategies to manipulate the system dynamics.

\section{Control versus Manipulation of the Earth's Climate System}

Operations to manipulate the weather and climate are mainly considered as intentional actions to influence natural processes within the ECS. Objectives of these actions are commonly formulated vaguely, in general terms and, consequently, expected results are fairly unpredictable and uncertain. However, since weather and climate manipulation 


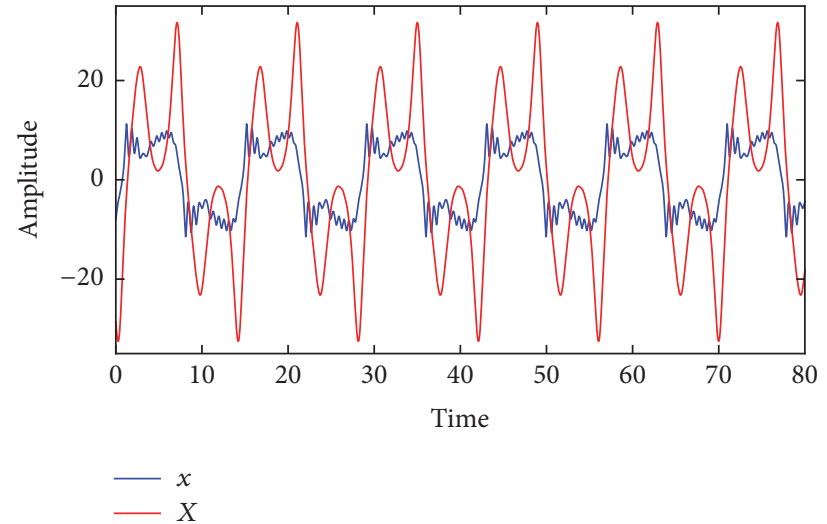

(a)

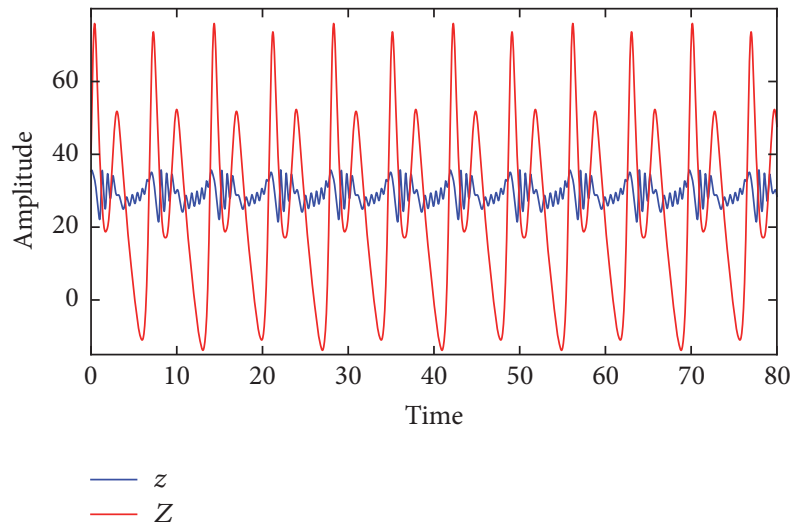

(b)

FIgURE 3: Fast ( $x$ and $z$ ) and slow $(X$ and $Z$ ) variables for the case of $c=0.8$.

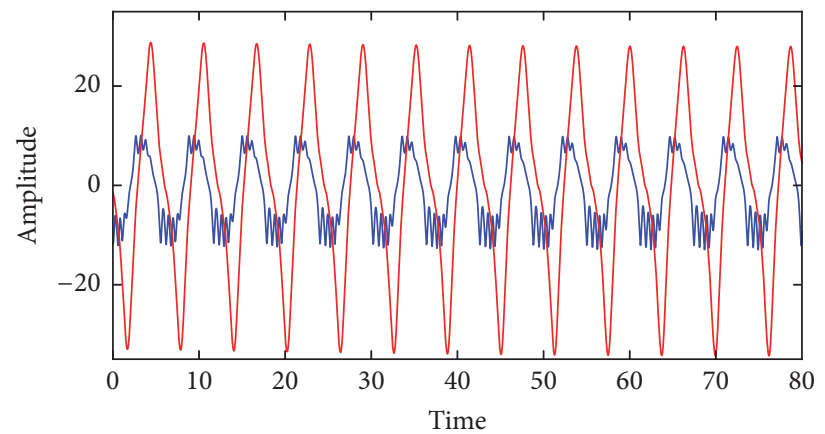

$-x$

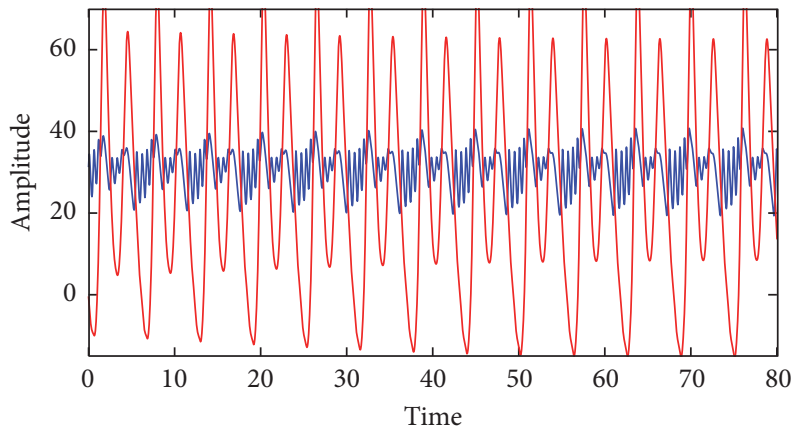

$-z$

(a)

(b)

FIGURE 4: Fast ( $x$ and $z$ ) and slow $(X$ and $Z$ ) variables for the case of $c=1.0$.

is a goal-directed process, we find it useful to formulate operations to manipulate weather and climate as the optimization problem within the framework of the optimal control theory (e.g., $[25,26])$. Then, instead of mathematically amorphous problem, we can obtain an optimal control problem for the ECS, which is formulated mathematically accurately. In this context the climate system and/or its components can be viewed as a control system and specially trained human operators will perform the role of controller.

The fundamental difference between weather and climate manipulation and control can be illustrated by the combination of block diagrams and transfer functions (e.g., [47, 48]). The transfer function of a system is simply the ratio of the Laplace transform of the system output to the Laplace transform of the system input assuming initial conditions are equal to zero. This approach used, for example, in signal processing, telecommunication, and control theory allows deriving the transfer function of complex system by pure algebraic operations. Transfer functions are applied for the study of linear, time invariant systems. Of cause, models used in weather and climate simulations are extremely complex and nonlinear. However, a number of properties of nonlinear systems can be explored using the assumption that for small perturbations the system is approximately linear. Figure 6 shows the block diagram for weather and climate manipulation operations. The signal $Z(s)$ fed to the system is a sum of state variables $X(s)$ and man-made perturbations $W(s)$, which serve as intentional actions to manipulate the weather and climate. The outcome of these intentional actions strongly depends on spatiotemporal distribution and magnitude of man-made perturbations. The determination of these perturbation parameters is performed heuristically, with the use of expert estimations. Therefore, the results of weather and climate manipulation are very uncertain and unpredictable. The transfer function for weather and climate manipulation operations is expressed as follows:

$$
T(s) \equiv \frac{Y(s)}{X(s)}=G(s)\left[1+\frac{W(s)}{X(s)}\right]
$$

Note that for climate manipulation $W(s) / X(s) \ll 1$, while for weather modification the ratio between $X(s)$ and $W(s)$ can vary. 


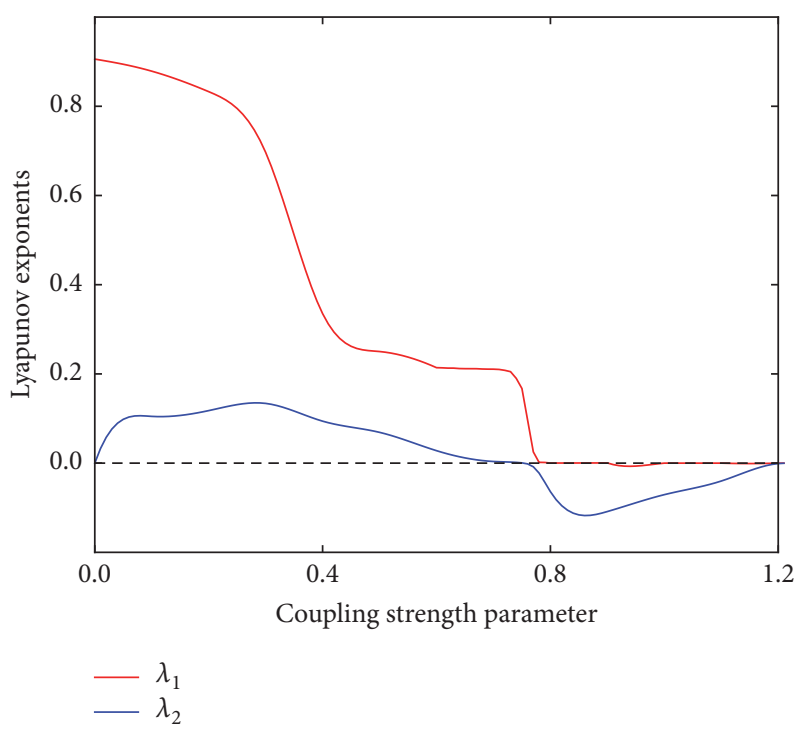

FIgURE 5: Two largest Lyapunov exponents.

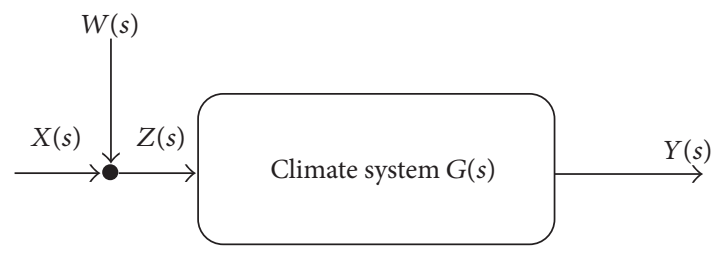

FIGURE 6: Block diagram for weather and climate manipulation.

The block diagram for weather and climate control is shown in Figure 7, which reflects both open-loop and closedloop control concepts. A controller is the essential integral part of control system. Since the atmosphere and other climate system components have a large number of positive and negative feedback mechanisms, we present the transfer function for weather and climate control operations taking into account closed-loop concept:

$$
T_{c}(s) \equiv \frac{Y(s)}{X(s)}=\frac{G(s)[1+C(s)]}{1+G(s) C(s) H(s)} .
$$

The transfer function technique is a very helpful tool to analyse feedback mechanisms in the ECS, providing important information relevant to the elaboration of climate control systems (e.g., [49-51]).

The ECS refers to a class of the distributed parameter systems, which have great difficulty not only in controlling but even in studying, simulating, and analysing. The reason is that mathematical models of the atmospheric and climate processes in spite of their complexities are not sufficiently accurate and adequate; therefore natural climate processes are not really well identified as control systems. To date, the control problem for the ECS processes remains poorly studied due to its immense complexity and originality. To develop a general theoretical framework for optimal control of the ECS we should to take the following into consideration.

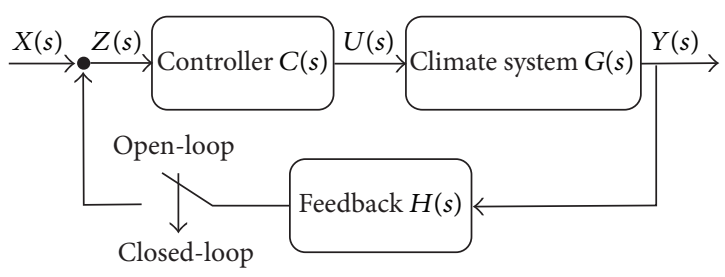

FIGURE 7: Blog diagram for feedback climate and weather control.

(i) The ECS is a spatially distributed system; consequently the control actions for manipulating the ECS should also be distributed in space; however, the implementation of such controls is faintly developed.

(ii) Processes in the ECS possess enormous energy potential. It is unlikely that we are able to provide control actions whose energy is comparable to the energy of natural climate processes. However, many processes in the ECS are unstable (or metastable) under almost regular conditions. Therefore, infinitesimal perturbations caused by small external forcing may lead to significant changes in the system dynamics. If the system is unstable, the energy barrier is minimal to change qualitatively the system behavior. The identification of sensitive points in which components of the ECS are unstable (metastable) is a critical issue for the development of methods and technique to control and manipulate the ECS processes.

(iii) Large spatiotemporal scales and huge energy of climate-driven processes impose rigorous requirements for the accuracy and reliability of control systems, since even insignificant errors in control actions can lead to disastrous consequences.

(iv) Processes in the ECS are interconnected and interdependent; therefore man-made changes in the dynamics of some processes can result in unpredictable and uncontrollable changes in the entire climate system.

(v) Control actions to perform geoengineering and weather modification operations must be physically feasible and practically executable.

Since we are going to explore the weather and climate manipulation as an optimal control problem, let us consider an abstract dynamical system of the form

$$
\begin{aligned}
& \dot{x}=f(x(t), u(t)) \\
& \quad x \in X \subseteq \mathbb{R}^{n}, u \in U \subset \mathbb{R}^{m}, t \in[0, T] \subset \mathbb{R}^{+}
\end{aligned}
$$

with the initial conditions

$$
x(0)=x_{0} .
$$

Suppose that the state vector $x$ belongs to the class of continuously differentiable functions $C^{1}([0, T])$ and the control vector $u$ belongs to the class of piecewise continuous functions $\widehat{C}([0, T])$. We can assume that control vector depends 
on the state of a system $u(t)=g(t, x(t))$, which means that system (6) is a closed-loop control system, representing the ECS. Suppose also that system (6) is controllable and control vector belongs to the set of admissible controls $u \in \mathcal{U} \subset U$. It is necessary to emphasize that the set of admissible controls $\mathcal{U}$ is determined on the foundation of physical feasibility and technical implementability of methods of weather and climate control. The optimal control aim is to synthesize the control law that ensures the accomplishment of the control objective. Formally an optimal control problem statement can be written as follows.

Find the control vector $u^{*}:[0, T] \mapsto \mathcal{U} \subset U$ and the orbit $x^{*}:[0, T] \mapsto \mathscr{X} \subset X$, which is generated by $u^{*}$, such that the given performance index (cost function) $\mathscr{J}(x, u)$ reaches a minimum (maximum) value; that is,

$$
\begin{aligned}
\mathscr{J}(x, u)= & f\left(t_{0}, x\left(t_{0}\right), T, x(T)\right) \\
& +\int_{0}^{T} \mathscr{F}(x(t), u(t), t) d t \longrightarrow \min .
\end{aligned}
$$

An integrand of the performance index $\mathscr{F}: \mathscr{X} \times \mathcal{U} \rightarrow$ $\mathbb{R}$. We denote by $\mathscr{X}$ a set of state variables on which the cost function is defined. Equation (6) represents the dynamic constraints. Performance index (8) corresponds to the Bolza problem, while the problems of Mayer and Lagrange are its special cases. The formulation of cost function is not trivial and depends on the problem under consideration. Unfortunately, there are no universal approaches regarding how the performance index can be specified. Optimal control problem (8) is nonlinear and can be solved by Pontryagin's maximum principle, dynamical programming, or classical approaches of the variational calculus.

As an example, consider an optimal control problem for the large-scale atmospheric dynamics that is characterized by the slowly moving Rossby waves. These planetary-scale waves strongly influence weather conditions over large geographical regions. Under some assumptions the Rossby wave dynamics at the so-called equivalent-barotropic level of the atmosphere can be described by the following vorticity equation $[52,53]$ :

$$
\partial_{t} \zeta+u \partial_{x}(\zeta+f)+v \partial_{y}(\zeta+f)=f\left(\frac{A}{P}\right) \omega_{0}
$$

where $u$ and $v$ are the components of wind velocity in the directions $x$ and $y$, respectively, $f$ is the Coriolis parameter, $\zeta=\partial_{x} v-\partial_{y} u$ is the vertical component of the relative vorticity, $P=10^{5} \mathrm{~Pa}$ is a "standard" surface pressure, $A(\xi)$ is an empirical function used to describe the vertical variation of the horizontal wind velocity, $\xi=p / P$ is a normalized pressure that plays the role of vertical coordinate, and $\omega_{0}$ is a vertical velocity at the level $\xi=1$, which corresponds to the earth's surface. Equation (9) is a forced barotropic vorticity equation since its right-hand side is nonzero. The forcing term is determined by the vertical motion at lower boundary $\omega_{0}$. The natural forcing of the atmospheric Rossby waves is of thermal and orographic origin. These two mechanisms are described by the right-hand side term in (9), that is, by the vertical velocity $\omega_{0}$. Therefore, this velocity can be considered as a control variable. Equation (9) can be transformed into the inhomogeneous Helmholtz equation [52]:

$$
\nabla^{2} q-\left(\frac{1}{L_{0}^{2}}\right) q=F
$$

Here $q=\partial \psi / \partial t$, where $\psi=\Phi / f$ is a geostrophic stream function and $\Phi$ is the geopotential; $1 / L_{0}^{2}=A R_{c} f / P$ is a coefficient that implicitly depends on $\omega_{0} ; R_{c}$ is a correlation coefficient that describes statistical relationship between the surface pressure and the mid-tropospheric geopotential; $F=-J\left(\psi, \nabla^{2} \psi+f\right)$, where $J(a, b)=(\partial a / \partial x)(\partial b / \partial y)-$ $(\partial a / \partial y)(\partial b / \partial x)$ is Jacobian.

Equation (10) is considered in a closed domain $D$ of the $x y$-plane with a piecewise continuous boundary $\partial D$. The boundary curve in parametric form is represented as $x=\gamma_{1}(\sigma)$ and $y=\gamma_{2}(\sigma)$, where $\gamma_{1}$ and $\gamma_{2}$ are continuous functions of the parameter $\sigma$. Suppose that the coordinate origin coincides with the North Pole; then $x=r \cos \vartheta$, and $y=r \sin \vartheta$, where $(r, \vartheta)$ is a polar coordinate of a generic point on the circle. The solution to (10) is defined on the temporal interval $[0, T]$. Specifying $\psi=$ const along the boundary $\partial D$ and introducing the new dependent variable $z=(\partial \psi / \partial t) \delta t$, where $\delta t$ is the time step of numerical integration, one can obtain the Dirichlet problem:

$$
\begin{aligned}
\nabla^{2} z-\left(\frac{1}{L_{0}^{2}}\right) z & =G \quad \text { in } D \\
z & =0 \text { on } \partial D
\end{aligned}
$$

where $G=F \delta t$. Problem (11) is solved numerically to obtain the stream function

$$
\psi(\delta t)=\psi_{0}+\left.z\right|_{t=0},
$$

where $\psi_{0}$ is the initial condition. Then one can calculate the forecast of geopotential field $\Phi=f \cdot \psi(\delta t)$, which characterizes the dynamics of Rossby waves. Note that the forward difference is applied on the first time step and then a central difference can be employed.

Consider the control system characterized by the state variable $z=z(x, y)$ and control variable $u=u(x, y)$, taking into account (11):

$$
\begin{aligned}
\nabla^{2} z-\left(\frac{1}{L_{0}^{2}}\right) z+u & =G \quad \text { in } D \\
z=0 & \text { on } \partial D .
\end{aligned}
$$

Note that the control $u$ represents the additional vertical velocity at the earth's surface. Let us define the performance index as follows:

$$
\mathscr{J}(x, u)=\frac{1}{2} \iint_{D}\left[\left(z-z^{*}\right)^{2}+\eta u^{2}\right] d D,
$$


where the pair $(z, u)$ satisfies (13) and $z^{*}$ is the desired spatial distribution of $z$ in the domain $D$. The optimal control problem is formulated as follows:

Find the control $u^{*} \in \mathcal{U}$ and the corresponding system state $z^{*} \in \mathscr{Z}$ such that cost function (14) is minimized.

Here $\mathcal{U}$ is a set of admissible controls and $\mathscr{Z}$ is a set of some additional constraints on the state variable $z$. From the standpoint of atmospheric physics, the control problem aims to manipulate the phase speed of Rossby waves by changing the vertical velocity at the earth's surface. Indeed the linearization of the vorticity equation around the unperturbed westerly zonal flow $u_{0}$ and then representation of the solution to the linearized equation in the form of plane waves

$$
\psi^{\prime}(x, y, t)=\Re \mathrm{e}\left[\Psi_{0} e^{i k(x-c t)}\right] \cos l y
$$

give the following expression for the phase speed of Rossby waves:

$$
c=\frac{u_{0}\left(k^{2}+l^{2}\right)-\left(\beta-u_{0}^{\prime \prime}\right)}{\left(k^{2}+l^{2}\right)+\left(1 / L_{0}^{2}\right)},
$$

where $\Psi_{0}$ is the amplitude of perturbation of stream function $\psi^{\prime}, k$ and $l$ are the zonal and meridional wavenumbers, $\beta=$ $\partial f / \partial y$ is the Rossby parameter, and $u_{0}^{\prime \prime}=d^{2} u_{0} / d y^{2}$. The latest formula shows that the vertical structure of the atmosphere via the term $1 / L_{0}^{2}$ contributes to the dispersion properties of Rossby waves. Meanwhile, the term $1 / L_{0}^{2}$ depends on the correlation coefficient $R_{c}$, which relates the vertical velocity $\omega_{0}$ with the stream function at the equivalent-barotropic level of the atmosphere. Consequently, the phase velocity of Rossby waves is in a function of $\omega_{0}$.

By introducing new dependent and parametric variables (13) can be written in the normal form [54]:

$$
\begin{aligned}
\frac{\partial z_{1}}{\partial x}-z_{2} & =0, \\
\frac{\partial z_{1}}{\partial y}-z_{3} & =0, \\
\frac{\partial z_{2}}{\partial x}-\chi_{1} & =0, \\
\frac{\partial z_{2}}{\partial y}-\chi_{2} & =0, \\
\frac{\partial z_{3}}{\partial x}-\chi_{2} & =0, \\
\frac{\partial z_{3}}{\partial y}+\chi_{1}+u-\left(\frac{1}{L_{0}^{2}}\right) z_{1}-G & =0 .
\end{aligned}
$$

Adjoin (17) to the cost function (14) with spatial-varying Lagrange multipliers $\lambda_{1}, \lambda_{2}, \lambda_{3}, \mu_{1}, \mu_{2}$, and $\mu_{3}$ :

$$
\begin{aligned}
\mathscr{J}_{A} & =\iint_{D}\left\{\frac{1}{2}\left[\left(z_{1}-z^{*}\right)^{2}+\eta u^{2}\right]+\lambda_{1}\left(z_{2}-\frac{\partial z_{1}}{\partial x}\right)\right. \\
& +\mu_{1}\left(z_{3}-\frac{\partial z_{1}}{\partial y}\right)+\lambda_{2}\left(\chi_{1}-\frac{\partial z_{2}}{\partial x}\right) \\
& +\mu_{2}\left(\chi_{2}-\frac{\partial z_{2}}{\partial y}\right)+\lambda_{3}\left(\chi_{2}-\frac{\partial z_{3}}{\partial x}\right) \\
& \left.+\mu_{3}\left(-\chi_{1}-u+\frac{1}{L_{0}^{2}}+G-\frac{\partial z_{3}}{\partial y}\right)\right\} d D .
\end{aligned}
$$

The Hamiltonian $\mathscr{H}$ associated with the optimal control problem is of the form

$$
\begin{aligned}
\mathscr{H}= & \frac{1}{2}\left[\left(z_{1}-z^{*}\right)^{2}+\eta u^{2}\right]+\lambda_{1} z_{2}+\lambda_{2} \chi_{1}+\lambda_{3} \chi_{2} \\
& +\mu_{1} z_{3}+\mu_{2} \chi_{2}+\mu_{3}\left(-\chi_{1}-u+\frac{1}{L_{0}^{2}} z_{1}+G\right) .
\end{aligned}
$$

The augmented cost function $\mathscr{J}_{A}$ is then rewritten as

$$
\begin{aligned}
\mathscr{J}_{A} & =\iint_{D}\left(\mathscr{H}-\lambda_{1} \frac{\partial z_{1}}{\partial x}-\lambda_{2} \frac{\partial z_{2}}{\partial x}-\lambda_{3} \frac{\partial z_{3}}{\partial x}-\mu_{1} \frac{\partial z_{1}}{\partial y}\right. \\
- & \left.\mu_{2} \frac{\partial z_{2}}{\partial y}-\mu_{3} \frac{\partial z_{3}}{\partial y}\right) d D
\end{aligned}
$$

or in the vector form

$$
\mathscr{J}_{A}=\iint_{D}\left(\mathscr{H}-\lambda \frac{\partial z}{\partial x}-\mu \frac{\partial z}{\partial y}\right) d D
$$

where $z=\left(z_{1}, z_{2}, z_{3}\right), \lambda=\left(\lambda_{1}, \lambda_{2}, \lambda_{3}\right)$, and $\mu=\left(\mu_{1}, \mu_{2}, \mu_{3}\right)$. By adding and subtracting the term $z(\partial \lambda / \partial x+\partial \mu / \partial y)$ to the integrand of the latest equation, the augmented cost function $\mathcal{F}_{A}$ takes the form

$$
\begin{aligned}
\mathscr{F}_{A}= & \iint_{D}\left[\mathscr{H}+\left(\frac{\partial \lambda}{\partial x}+\frac{\partial \mu}{\partial y}\right) z\right] d D \\
& -\iint_{D}\left[\frac{\partial}{\partial x}(\lambda z)+\frac{\partial}{\partial y}(\mu z)\right] d D .
\end{aligned}
$$

Using Green's theorem, the second integral in this expression becomes a line integral, and then $\mathscr{J}_{A}$ can be rewritten as

$$
\begin{aligned}
\mathscr{J}_{A}= & \iint_{D}\left[\mathscr{H}+\left(\frac{\partial \lambda}{\partial x}+\frac{\partial \mu}{\partial y}\right) z\right] d D \\
& +\int_{\partial D}\left[\lambda \gamma_{2}^{\prime}(\sigma)-\mu \gamma_{1}^{\prime}(\sigma)\right] z d \sigma .
\end{aligned}
$$

The first variation in $\mathscr{J}_{\mathrm{A}}$ caused by variations in $u$ and $z$ is

$$
\begin{aligned}
\delta \mathscr{F}_{A}= & \int_{D}\left[\left(\frac{\partial \mathscr{H}}{\partial z}+\frac{\partial \lambda}{\partial x}+\frac{\partial \mu}{\partial y}\right) \delta z+\frac{\partial \mathscr{H}}{\partial u_{1}}\right] d D \\
& +\int_{\partial D}\left[\lambda \gamma_{2}^{\prime}(\sigma)-\mu \gamma_{1}^{\prime}(\sigma)\right] \delta z d \sigma,
\end{aligned}
$$


where $u_{1}=\left(u, \chi_{1}, \chi_{2}\right)$. We can select both $\lambda$ and $\mu$ to cause the first term in parentheses in the double integral to vanish:

$$
\frac{\partial \lambda}{\partial x}+\frac{\partial \mu}{\partial y}=-\frac{\partial \mathscr{H}}{\partial z}
$$

The function $z_{1}$ does not change along the boundary $\partial D\left(z_{1}=\right.$ 0 ); therefore its variation $\delta z_{1}=0$ on $\partial D$. Functions $z_{2}$ and $z_{3}$ are not defined on the boundary $\partial D$; therefore we can require the relationship $\lambda \gamma_{2}^{\prime}(\sigma)-\mu \gamma_{1}^{\prime}(\sigma)=0$ to hold along $\partial D$. Then in polar coordinate we can obtain the boundary conditions for (17):

$$
\begin{aligned}
& \lambda_{2} \cos \vartheta+\mu_{2} \sin \vartheta=0, \\
& \lambda_{3} \cos \vartheta+\mu_{3} \sin \vartheta=0 .
\end{aligned}
$$

Then the first variation in $\mathscr{J}_{A}$ becomes

$$
\delta \mathscr{J}_{A}=\int_{D}\left(\frac{\partial \mathscr{H}}{\partial u_{1}} \delta u_{1}\right) d D
$$

At the stationary point $\delta \mathscr{J}_{A}=0$, which can be achieved if

$$
\frac{\partial \mathscr{H}}{\partial u_{1}}=0
$$

Equations (25) and (28) with boundary conditions (13) and (26) are the necessary conditions of optimality that can be represented in the expanded form as

$$
\begin{aligned}
\frac{\partial \lambda_{1}}{\partial x}+\frac{\partial \mu_{1}}{\partial y} & =-\left(z_{1}-z^{*}\right)-\frac{\mu_{3}}{L_{0}^{2}} \\
\frac{\partial \lambda_{2}}{\partial x}+\frac{\partial \mu_{2}}{\partial y} & =-\lambda_{1}, \\
\frac{\partial \lambda_{3}}{\partial x}+\frac{\partial \mu_{3}}{\partial y} & =-\mu_{1}, \\
\eta u-\mu_{3} & =0 \\
\lambda_{2}-\mu_{3} & =0 \\
\lambda_{3}+\mu_{2} & =0 .
\end{aligned}
$$

Thus, to find the control $u$ that minimizes cost function (14) one must solve the set of 12 partial differential equations (17) and (29) with 12 unknowns and with specified boundary conditions:

$$
\begin{aligned}
& \left.z_{1}\right|_{\partial D}=0, \\
& \left.\lambda_{2}\right|_{\partial D}=\left.\mu_{2}\right|_{\partial D}=0 .
\end{aligned}
$$

For particular case of the optimal control problem $(\eta=$ 0 ) we obtain $\mu_{3}=\lambda_{2}=0$ and the necessary conditions for optimality take the form

$$
\begin{aligned}
\frac{\partial \lambda_{1}}{\partial x}+\frac{\partial \mu_{1}}{\partial y} & =-\left(z_{1}-z^{*}\right), \\
\frac{\partial \mu_{2}}{\partial y} & =-\lambda_{1}, \\
\frac{\partial \lambda_{3}}{\partial x} & =-\mu_{1}, \\
\lambda_{3}+\mu_{2} & =0 .
\end{aligned}
$$

Since $\lambda_{3}=-\mu_{2}$, the second and third equations in (31) are similar to the first two equations of system (17); therefore, the following assumptions can be made [55]:

$$
\begin{aligned}
& \lambda_{1}=\frac{z_{3}}{\alpha}, \\
& \mu_{1}=\frac{z_{2}}{\alpha}, \\
& \lambda_{3}=-\mu_{2}=\frac{z_{1}}{\alpha},
\end{aligned}
$$

where $\alpha$ is an arbitrary constant. These assumptions are compatible with boundary conditions (30). The substitution of (32) into the first equation of system (32) gives

$$
\left(\frac{\partial^{2} z_{1}}{\partial x \partial y}-\frac{\partial^{2} z_{1}}{\partial x \partial y}\right)=-\alpha\left(z_{1}-z^{*}\right) .
$$

Therefore, we have that $\alpha\left(z_{1}-z^{*}\right)=0$ in the domain $D$. It follows that the cost function attains its absolute minimum under constraint (13). In order to find the optimal control $u$ that minimizes the cost function, one should solve the following problem in the domain $D$ :

$$
\begin{aligned}
u & =-\nabla^{2} z^{*}-\left(\frac{1}{L_{0}^{2}}\right) z^{*}+G_{0}, \\
\left.z^{*}\right|_{\partial D} & =0 .
\end{aligned}
$$

The Rossby waves propagate westward relative to the mean quasi-zonal flow. If the optimal control objective is to reach the stationarity of these waves with respect to the surface by manipulating the lower boundary vertical velocity $\omega_{0}$, we should solve the following equation to obtain the optimal control: $u=G_{0}$, since in this case $z^{*} \equiv \partial \psi / \partial t=0$.

Thus, we mathematically accurately proved the existence of the absolute minimum of cost function (14) under constraint (13). In the problem considered above, the vertical velocity at the earth's surface was chosen as control variable. Generally, however, the determination of feasible control variables is not a trivial problem. The "instability-sensitivity" approach suggested in [56] can serve as one of the possible ways to substantiate the choice of control variables in weather and climate control. This method is theoretically based on the fact that some processes in the ECS are inherently unstable 
under almost regular conditions. Since in mathematical models used in climate studies control actions that manipulate the weather and climate can be expressed via variations in model parameters that act as controls, sensitivity analysis of atmospheric and climate models allows the determination of parameters that can be considered as controls. Using mathematical models that describe various types of natural instability phenomena, one can find the necessary conditions for instability and estimate the influence of various model parameters on the development of instability. The obtained results provide very important information that can be taken into account in designing control systems for atmospheric and climate processes.

\section{Concluding Remarks}

Since the ECS possesses a full spectrum of properties of complex adaptive systems, the exploration of the atmosphere and other components of the ECS as complex adaptive systems allows the consideration of weather and climate manipulation as an optimization problem within the scope of the optimal control theory. However, the complexity of the ECS makes the control problem extremely difficult to solve. In this paper, the necessary conditions of optimality are derived for control of the large-scale atmospheric dynamics. This demonstrates that even a relatively simplified control problem for the atmospheric Rossby waves requires significant efforts to obtain the solution. The study of the ECS as a complex adaptive system, the exploration of its feedback mechanisms, and interactions between climate system components represent the most appropriate approach to estimate the effectiveness and feasibility of potential methods to control the weather and climate. The use of "instability-sensitivity" approach allows the determination of feasible control variables.

In our subsequent papers we intend to show how the optimal control theory makes it possible to obtain the mathematically accurate solution to the problem of weather and climate manipulation.

\section{Competing Interests}

The author declares that they have no competing interests.

\section{References}

[1] R. N. Hoffman, "Controlling the global weather," Bulletin of the American Meteorological Society, vol. 83, no. 2, pp. 241-248, 2002.

[2] J. Shepherd, K. Caldeira, J. Haigh et al., "Geoengineering the climate: science, governance and uncertainty," Royal Society Report 10/09, RS1636, The Royal Society, London, UK, 2009.

[3] IPCC, "Climate change 2013: the physical science basis," in Contribution of Working Group I to the Fifth Assessment Report of the Intergovernmental Panel on Climate Change, T. F. Stocker, D. Qin, G.-K. Plattner et al., Eds., Cambridge University Press, Cambridge, UK, 2013.

[4] K. E. Trenberth, Climate System Modeling, Cambridge University Press, Cambridge, UK, 1992.
[5] W. M. Washington and C. L. Parkinson, An Introduction to Three-Dimensional Climate Modeling, University Science Books, Sausalito, Calif, USA, 2005.

[6] W. H. Ruddiman, Earth's Climate: Past and Future, W. H. Freeman and Company, New York, NY, USA, 2008.

[7] J. E. Oliver and H. A. Bridgman, The Global Climate System: Patterns, Processes, and Teleconnections, Cambridge University Press, Cambridge, UK, 2014.

[8] R. G. Barry and E. A. Hall-McKim, Essentials of the Earth's Climate System, Cambridge University Press, Cambridge, UK, 2014.

[9] S. R. Weart, The Discovery of Global Warming, Harvard University Press, Cambridge, Mass, USA, 2014.

[10] A. S. Dennis, Changing of Weather by Cloud Seeding, Academic Press, New York, NY, USA, 1980.

[11] M. Ćurić, D. Janc, and V. Vučković, "Cloud seeding impact on precipitation as revealed by cloud-resolving mesoscale model," Meteorology and Atmospheric Physics, vol. 95, no. 3-4, pp. 179193, 2007.

[12] D. L. Mitchell and W. Finnegan, "Modification of cirrus clouds to reduce global warming," Environmental Research Letters, vol. 4, no. 4, Article ID 045102, 2009.

[13] X. Guo, D. Fu, X. Li et al., "Advances in cloud physics and weather modification in China," Advances in Atmospheric Sciences, vol. 32, no. 2, pp. 230-249, 2015.

[14] I. Colbeck, "The development of fog intensive dispersal operation," in Aerosol Science and Technology: History and Reviews, D. S. Ensor, Ed., pp. 367-375, RTI Press, Research Triangle Park, NC, USA, 2011.

[15] M. Garstang, R. Bruintjes, R. Serafin et al., "Weather modification: finding common ground," Bulletin of the American Meteorological Society, vol. 86, no. 5, pp. 647-655, 2005.

[16] S. D. Aberson and J. L. Franklin, "Impact on Hurricane Track and Intensity Forecasts of GPS Dropwindsonde Observations from the First-Season Flights of the NOAA Gulfstream-IV Jet Aircraft," Bulletin of The American Meteorological Society, vol. 80, no. 3, pp. 421-427, 1999.

[17] J. R. Fleming, Fixing the Sky: The Checkered History of Weather and Climate Control, Columbia University Press, New York, NY, USA, 2010.

[18] L. Bengtsson, "Geo-engineering to confine climate change: is it at all feasible?" Climatic Change, vol. 77, no. 3-4, pp. 229-234, 2006.

[19] P. J. Crutzen, "Albedo enhancement by stratospheric sulfur injections: a contribution to resolve a policy dilemma?" Climatic Change, vol. 77, no. 3-4, pp. 211-220, 2006.

[20] T. M. L. Wigley, "A combined mitigation/geoengineering approach to climate stabilization," Science, vol. 314, no. 5798, pp. 452-454, 2006.

[21] A. Robock, A. Marquardt, B. Kravitz, and G. Stenchikov, "Benefits, risks, and costs of stratospheric geoengineering," Geophysical Research Letters, vol. 36, no. 19, Article ID L19703, 2009.

[22] M. C. MacCracken, "On the possible use of geoengineering to moderate specific climate change impacts," Environmental Research Letters, vol. 4, no. 4, Article ID 045107, pp. 1-14, 2009.

[23] J. McClellan, D. W. Keith, and J. Apt, "Cost analysis of stratospheric albedo modification delivery systems," Environmental Research Letters, vol. 7, no. 3, Article ID 034019, 2012.

[24] T. Ming, R. De Richter, W. Liu, and S. Caillol, "Fighting global warming by climate engineering: is the Earth radiation 
management and the solar radiation management any option for fighting climate change?" Renewable and Sustainable Energy Reviews, vol. 31, pp. 792-834, 2014.

[25] D. V. Gaskarov, V. B. Kisselev, S. A. Soldatenko, V. I. Strogonov, and R. M. Yusupov, An Introduction to Geophysical Cybernetics and Environmental Monitoring, St. Petersburg State University, St Petersburg, Russia, 1998.

[26] S. Soldatenko and R. Yusupov, "On the possible use of geophysical cybernetics in climate manipulation (Geoengineering) and weather modification," WSEAS Transactions on Environment and Development, vol. 11, pp. 116-125, 2015.

[27] J. S. Barnett and R. G. Cameron, Introduction to Mathematical Control Theory, Oxford University Press, Oxford, UK, 1990.

[28] C. P. Summerhayes, Earth's Climate Evolution, Wiley-Blackwell, 2015.

[29] D. Meadows, Thinking in Systems: A Primer, Chelsea Green, White River Junction, Vt, USA, 2008.

[30] M. M. Waldrop, Complexity: The Emerging Science at the Edge of Order and Chaos, Simon \& Schuster, New York, NY, USA, 1993.

[31] C. Gros, Complex and Adaptive Dynamical Systems, SpringerVerlag, Berlin, Germany, 2008.

[32] J. H. Miller and E. Page, Complex Adaptive Systems: An Introduction to Computational Models of Social Life, Princeton University Press, Princeton, NJ, USA, 2009.

[33] H. A. Dijkstra, Nonlinear Climate Dynamics, Cambridge University Press, New York, NY, USA, 2013.

[34] A. Ruzmaikin, "Climate as a game of chance," Physics-Uspekhi, vol. 57, no. 3, pp. 280-291, 2014.

[35] F. Mezinger and A. Arakawa, Numerical Methods Used in Atmospheric Models, GARP Publications Series, no 17, vol. 2, WMO, 1979.

[36] L. M. Resler, "Edward N Lorenz's 1963 paper, 'Deterministic nonperiodic flow', in Journal of the Atmospheric Sciences, Vol 20, pages 130-141: Its history and relevance to physical geography," Progress in Physical Geography, vol. 40, no. 1, pp. 175-180, 2016.

[37] M. Peña and E. Kalnay, "Separating fast and slow modes in coupled chaotic systems," Nonlinear Processes in Geophysics, vol. 11, no. 3, pp. 319-327, 2004.

[38] L. Siqueira and B. Kirtman, "Predictability of a low-order interactive ensemble," Nonlinear Processes in Geophysics, vol. 19, no. 2, pp. 273-282, 2012.

[39] S. Soldatenko and D. Chichkine, "Correlation and spectral properties of a coupled nonlinear dynamical system in the context of numerical weather prediction and climate modeling," Discrete Dynamics in Nature and Society, vol. 2014, Article ID 498184, 16 pages, 2014.

[40] T. N. Palmer, "Extended-range atmospheric prediction and the Lorenz model," Bulletin-American Meteorological Society, vol. 74, no. 1, pp. 49-65, 1993.

[41] G. Boffetta, P. Giuliani, G. Paladin, and A. Vulpiani, "An extension of the Lyapunov analysis for the predictability problem," Journal of the Atmospheric Sciences, vol. 55, no. 23, pp. 34093416, 1998.

[42] B. Saltzman, "Finite amplitude free convection as an initial value problem-I," Journal of the Atmospheric Sciences, vol. 19, no. 4, pp. 329-341, 1962.

[43] C. Sparrow, The Lorenz Equations: Bifurcations, Chaos, and Strange Attractors, vol. 41 of Applied Mathematical Sciences, Springer, New York, NY, USA, 1982.
[44] M. G. Rosenblum, A. S. Pikovsky, and J. Kurths, "Phase synchronization of chaotic oscillators," Physical Review Letters, vol. 76, no. 11, pp. 1804-1807, 1996.

[45] R. Sausen, K. Barthel, and K. Hasselmann, "Coupled oceanatmosphere models with flux correction," Climate Dynamics, vol. 2, no. 3, pp. 145-163, 1988.

[46] A. S. Goudie and D. J. Cuff, Encyclopedia of Global Change: Environmental Change and Human Society, vol. 1, Oxford University Press, Oxford, UK, 2001.

[47] D. E. Seborg, T. F. Edgar, and D. A. Mellichamp, Process Dynamics and Control, John Wiley \& Sons, Hoboken, NJ, USA, 2003.

[48] C. Mei, "On teaching the simplification of block diagrams," International Journal of Engineering Education, vol. 18, no. 6, pp. 697-703, 2002.

[49] D. G. MacMynowski and E. Tziperman, "Testing and improving ENSO models by process using transfer functions," Geophysical Research Letters, vol. 37, no. 19, Article ID L19701, 2010.

[50] A. Jarvis and D. Leedal, “The Geoengineering Model Intercomparison Project (GeoMIP): a control perspective," Atmospheric Science Letters, vol. 13, no. 3, pp. 157-163, 2012.

[51] D. G. MacMartin, B. Kravitz, D. W. Keith, and A. Jarvis, "Dynamics of the coupled human-climate system resulting from closed-loop control of solar geoengineering," Climate Dynamics, vol. 43, no. 1-2, pp. 243-258, 2014.

[52] I. A. Kiebel, An Introduction to The Hydro-Dynamical Methods of Short Period Weather Forecasting, Pergamon Press, New York, NY, USA, 1963.

[53] J. R. Holton, An Introduction to Dynamic Meteorology, Elsevier, London, UK, 4th edition, 2004.

[54] K. A. Lurie, Applied Optimal Control Theory of Distributed Systems, Springer, New York, NY, USA, 1993.

[55] J.-L. Armand, Applications of the Theory of Optimal Control of Distributed Parameter Systems to Structural Optimization, NASA CR-2044, National Aeronautics and Space Administration, Washington, DC, USA, 1972.

[56] S. A. Soldatenko and R. M. Yusupov, "The determination of feasible control variables for geoengineering and weather modification based on the theory of sensitivity in dynamical systems," Journal of Control Science and Engineering, vol. 2016, Article ID 1547462, 9 pages, 2016. 


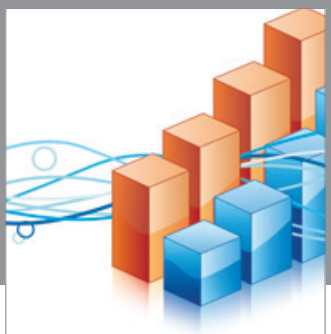

Advances in

Operations Research

vatem alat4

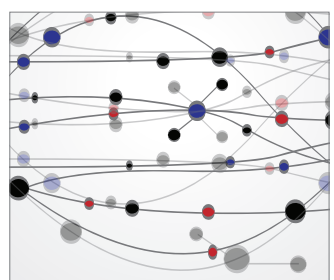

\section{The Scientific} World Journal
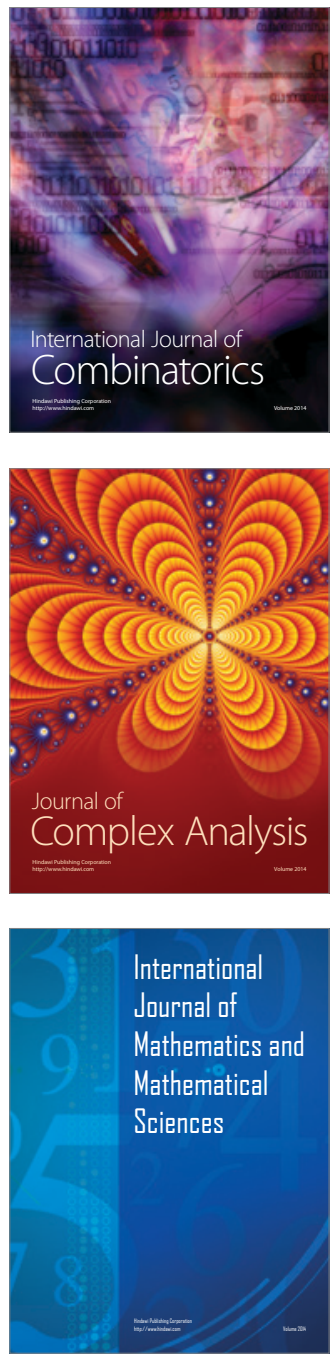
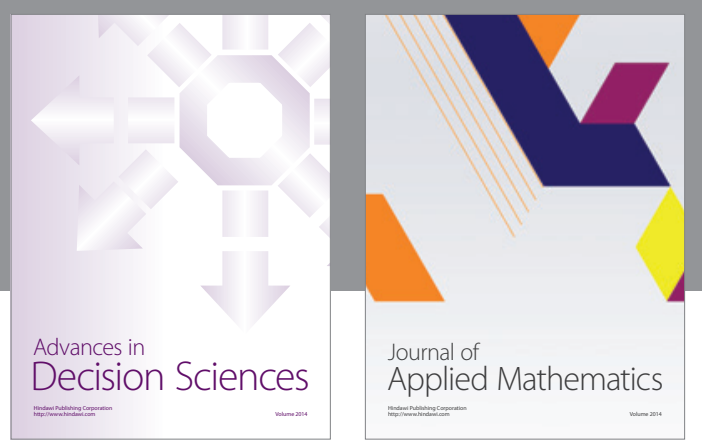

Algebra

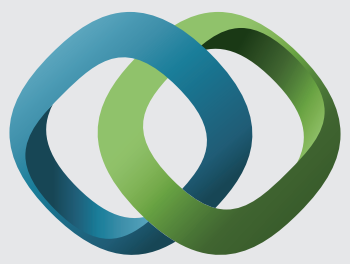

\section{Hindawi}

Submit your manuscripts at

https://www.hindawi.com
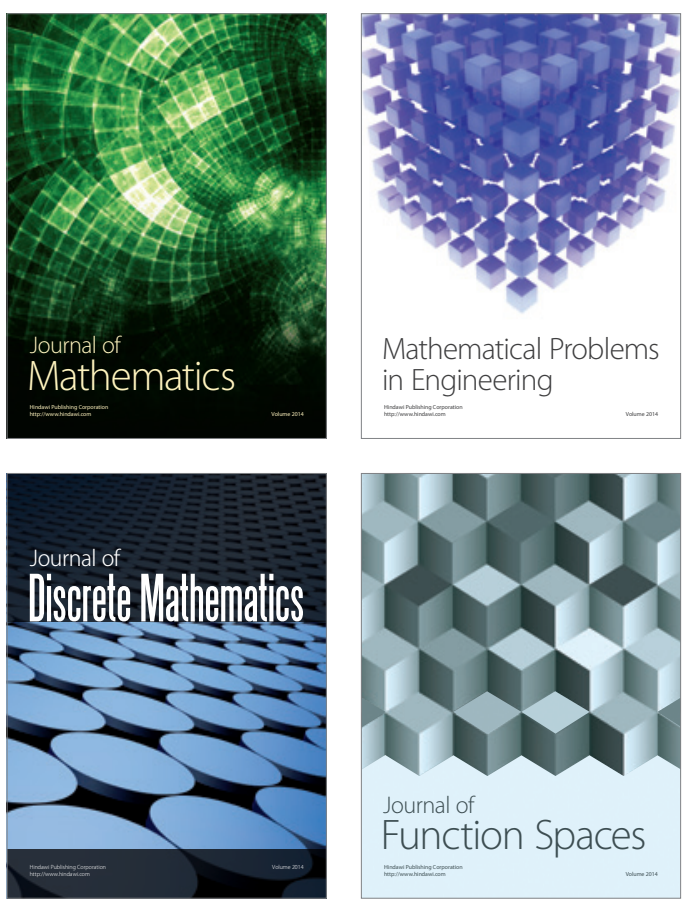

Mathematical Problems in Engineering
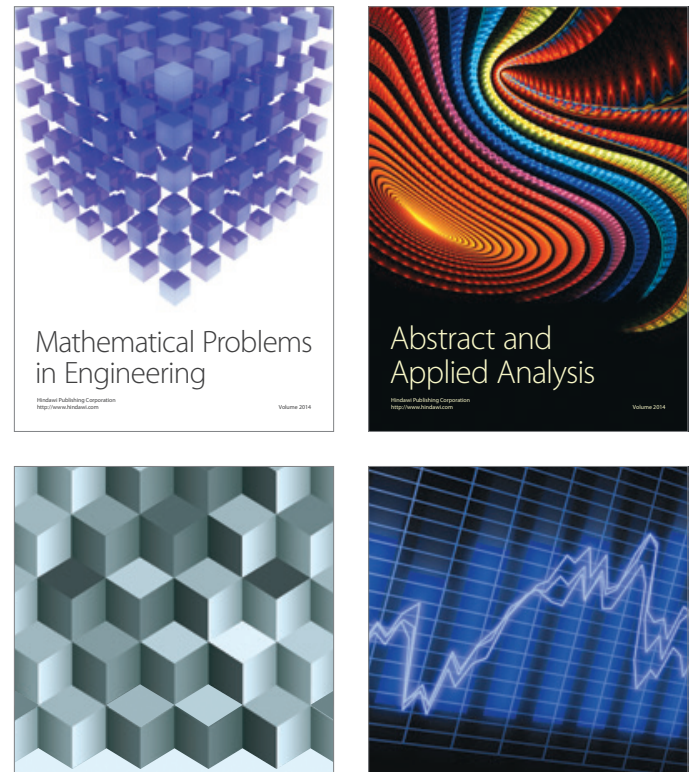

Journal of

Function Spaces

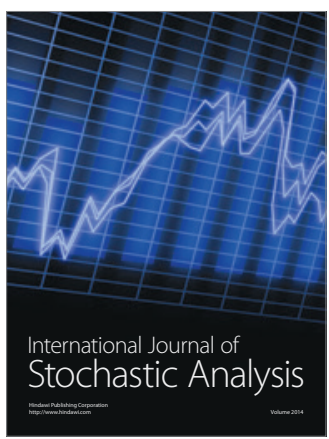

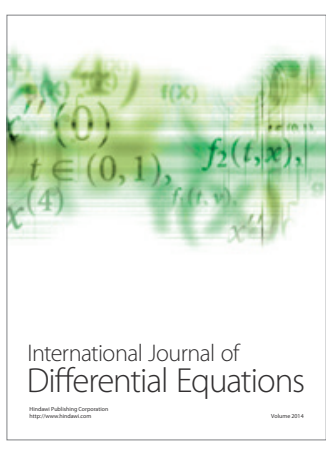
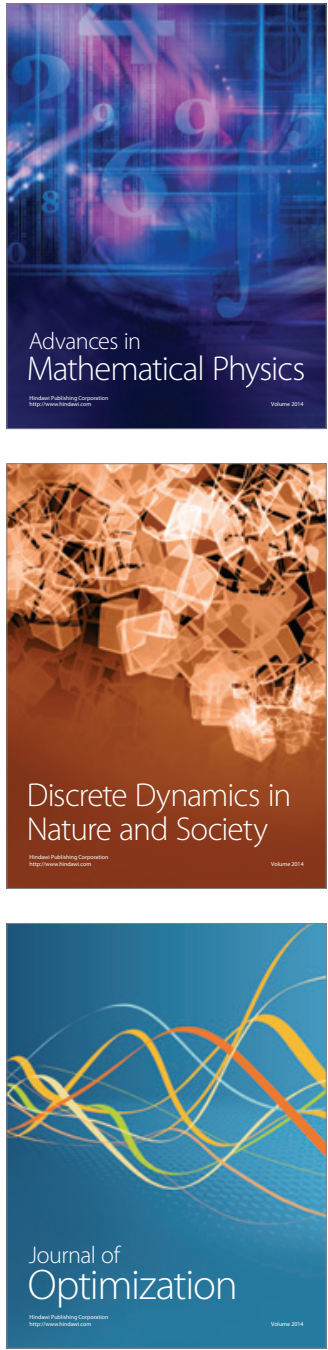